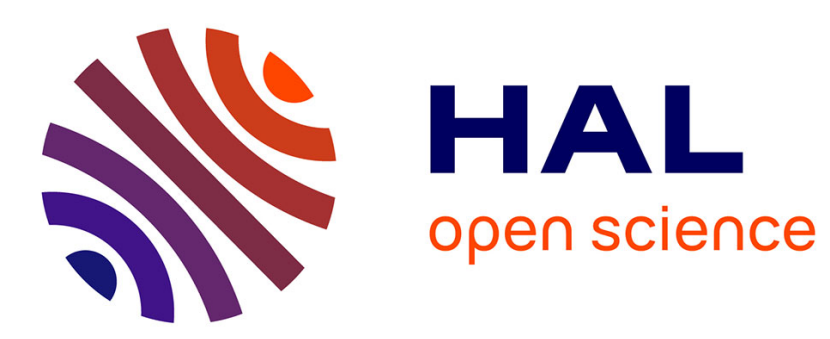

\title{
Photonic jet reconstruction for particle refractive index measurement by digital in-line holography
}

\author{
Matthias Sentis, Fabrice R. A. Onofri, Fabrice Lamadie
}

\section{To cite this version:}

Matthias Sentis, Fabrice R. A. Onofri, Fabrice Lamadie. Photonic jet reconstruction for particle refractive index measurement by digital in-line holography. Optics Express, 2017, 25 (2), pp.867-873. 10.1364/OE.25.000867.v001 . hal-01442106

\section{HAL Id: hal-01442106 https://hal.science/hal-01442106}

Submitted on 20 Nov 2019

HAL is a multi-disciplinary open access archive for the deposit and dissemination of scientific research documents, whether they are published or not. The documents may come from teaching and research institutions in France or abroad, or from public or private research centers.
L'archive ouverte pluridisciplinaire HAL, est destinée au dépôt et à la diffusion de documents scientifiques de niveau recherche, publiés ou non, émanant des établissements d'enseignement et de recherche français ou étrangers, des laboratoires publics ou privés. 


\title{
Photonic jet reconstruction for particle refractive index measurement by digital in-line holography
}

\author{
Matthias P.L. Sentis, ${ }^{1}$ FABrice R.A. ONOFRI, ${ }^{2}$ And Fabrice Lamadie ${ }^{1, *}$ \\ ${ }^{1}$ CEA, DEN, DTEC, SGCS, F-30207 Bagnols-sur-Cèze, France \\ ${ }^{2}$ Aix-Marseille Université, CNRS, IUSTI, UMR 7343, 13453 Marseille Cedex 13, France \\ *fabrice.lamadie@cea.fr
}

\begin{abstract}
A new and computationally efficient approach is proposed for determining the refractive index of spherical and transparent particles, in addition to their size and 3D position, using digital in-line holography. The method is based on the localization of the maximum intensity position of the photonic jet with respect to the particle center retrieved from the back propagation of recorded holograms. Rigorous electromagnetic calculations and experimental results demonstrate that for liquid-liquid systems and droplets with a radius $>30 \mu \mathrm{m}$, a refractive index measurement with a resolution inferior to $4 \times 10^{-3}$ is achievable, revealing a significant potential for the use of this method to investigate multiphase flows. The resolution for solid or liquid particles in gas is expected to be lower but sufficient for the recognition of particle material.
\end{abstract}

(C) 2016 Optical Society of America

OCIS codes: (090.1995) Digital holography; (120.3940) Metrology, (280.2940) Flow diagnostics, (290.5850) Scattering, particles

\section{References and links}

1. J. W. Goodman, Introduction to Fourier Optics, (McGraw-Hill Book Co. New York, 1968).

2. B. J. Thompson, "Holographic particle sizing techniques," Journal of Physics E: Scientific Instruments 7, 781 (1974).

3. X. Liang, A. Liu, C. Lim, T. Ayi, and P. Yap, "Determining refractive index of single living cell using an integrated microchip," Sensors and Actuators A: Physical 133, 349-354 (2007).

4. Y. Sung, N. Lue, B. Hamza, J. Martel, D. Irimia, R. R. Dasari, W. Choi, Z. Yaqoob, and P. So, "Threedimensional holographic refractive-index measurement of continuously flowing cells in a microfluidic channel," Physical review applied 1, 014002 (2014).

5. F. Koohyar, "Refractive Index and Its Applications," Journal of Thermodynamics \& Catalysis 4, 117 (2013).

6. D. Subedi, D. Adhikari, U. Joshi, H. Poudel, and B. Niraula, "Study of temperature and concentration dependence of refractive index of liquids using a novel technique," Kathmandu University Journal of Science, Engineering and Technology 2, 1-7 (2006).

7. C.-Y. Tan and Y.-X. Huang, "Dependence of refractive index on concentration and temperature in electrolyte solution, polar solution, nonpolar solution, and protein solution," Journal of Chemical \& Engineering Data 60, 2827-2833 (2015)

8. J. P. A. J. van Beeck, "Rainbow phenomena: development of a laser-based, non-intrusive technique for measuring droplet size, temperature and velocity," (1997).

9. F. Onofri, T. Girasole, G. Gréhan, G. Gouesbet, G. Brenn, J. Domnick, T. H. Xu, and C. Tropea, "PhaseDoppler Anemometry with the Dual Burst Technique for measurement of refractive index and absorption coefficient simultaneously with size and velocity," Particle \& particle systems characterization 13, 112-124 (1996).

10. S.-H. Lee, Y. Roichman, G.-R. Yi, S.-H. Kim, S.-M. Yang, A. van Blaaderen, P. van Oostrum, and D. G. Grier, "Characterizing and tracking single colloidal particles with video holographic microscopy," Opt Express 15, 18275-18282 (2007).

11. H. Shpaisman, B. J. Krishnatreya, and D. G. Grier, "Holographic microrefractometer," Appl. Phys. Lett. 101, $091102(2012)$.

12. A. Yevick, M. Hannel, and D. G. Grier, "Machine-learning approach to holographic particle characterization," Opt Express 22, 26884-26890 (2014).

13. M. P. Sentis, L. Bruel, S. Charton, F. R. Onofri, and F. Lamadie, "Digital in-line holography for the characterization of flowing particles in astigmatic optical systems," Opt Lasers Eng 88, 184-196 (2017). 
Matthias P. L. Sentis, Fabrice R. A. Onofri, and Fabrice Lamadie, "Photonic jet reconstruction for particle refractive index measurement by digital in-line holography," Opt. Express 25, 867-873 (2017) https://doi.org/10.1364/OE.25.000867

14. S. Lecler, Y. Takakura, and P. Meyrueis, "Properties of a three-dimensional photonic jet," Opt. Lett. 30, 2641$2643(2005)$.

15. J. A. Lock, C. L. Adler, and E. A. Hovenac, "Exterior caustics produced in scattering of a diagonally incident plane wave by a circular cylinder: semiclassical scattering theory analysis," J Opt Soc Am A 17, 1846-1856 (2000).

16. A. Heifetz, S.-C. Kong, A. V. Sahakian, A. Taflove, and V. Backman, "Photonic nanojets," Journal of computational and theoretical nanoscience 6, 1979-1992 (2009).

17. A. Itagi and W. Challener, "Optics of photonic nanojets," J Opt Soc Am A 22, 2847-2858 (2005).

18. A. Devilez, B. Stout, N. Bonod, and E. Popov, "Spectral analysis of three-dimensional photonic jets," Opt Express 16, 14200-14212 (2008).

19. P. Ferrand, J. Wenger, A. Devilez, M. Pianta, B. Stout, N. Bonod, E. Popov, and H. Rigneault, "Direct imaging of photonic nanojets," Opt Express 16, 6930-6940 (2008).

20. Y. E. Geints, E. Panina, and A. Zemlyanov, "Control over parameters of photonic nanojets of dielectric microspheres," Opt. Commun. 283, 4775-4781 (2010).

21. H. Ding, L. Dai, and C. Yan, "Properties of the 3D photonic nanojet based on the refractive index of surroundings," Chinese Optics Letters 8, 706-708 (2010).

22. F. Lamadie, L. Bruel, and M. Himbert, "Digital holographic measurement of liquid-liquid two-phase flows," Opt Lasers Eng 50, 1716-1725 (2012).

23. J. Sheng, E. Malkiel, and J. Katz, "Digital holographic microscope for measuring three-dimensional particle distributions and motions," Appl. Opt. 45, 3893-3901 (2006).

24. F. Lamadie and L. Bruel, "Processing method for near-field in-line holograms (Fresnel number $\geq 1$ )," Opt Lasers Eng 57, 130-137 (2014).

25. C. L. Adler, J. A. Lock, B. R. Stone, and C. J. Garcia, "High-order interior caustics produced in scattering of a diagonally incident plane wave by a circular cylinder," J Opt Soc Am A 14, 1305-1315 (1997).

26. M. P. Sentis, F. R. Onofri, L. Méès, and S. Radev, "Scattering of light by large bubbles: Coupling of geometrical and physical optics approximations," J Quant Spectrosc Radiat Transf, 8-18 (2015).

27. R. I. Masel, Chemical kinetics and catalysis (Wiley-Interscience New York, 2001).

\section{Introduction}

Non-intrusive tracking and characterization of micrometric to millimetric particles are key issues in various academic and industrial fields that can be addressed by several techniques. One such technique is digital in-line holography (DH), which has proved its relevance by providing a complete 3D recovery of the sizes and positions of a population of spherical particles with one data acquisition [1] using a simple and stable setup [2]. However, for many applications, it is also crucial to determine the composition of the particles. In the frame of linear optics, this can be achieved by measuring the refractive index of the particles. For example, the refractive index is used to detect variations in the chemical or structural properties of living cells $[3,4]$ or to determine the mixture fraction or temperature of droplets in chemical engineering applications [5-7].

The refractive index of droplets has been measured using optical techniques such as rainbow refractometry [8] and phase Doppler anemometry [9], but far less frequently using DH [10, 11]. With the latter approach $[10,11]$, the refractive index is obtained by comparing experimental holograms with full electromagnetic calculations performed using the Lorenz-Mie theory (LMT). Thus, the extension of this approach to large particles in free flow would require enormous amounts of computational resources [12].

In this paper, we propose a new and computationally efficient approach for determining the refractive index of spherical refracting particles using DH. The method is derived from the analysis [13] of the photonic jet generated by refracting particles when they are illuminated by a coherent light beam [14]. During the last decade, this optical near-field caustic [15] was extensively studied numerically [14, 16-18] and experimentally [16, 19]. Direct calculations have shown that the relative position of the maximum intensity of the photonic jet greatly depends on the optical properties of the particle material [20,21]. We propose to reconstruct the near field of the particles using DH and to use a simple geometrical optics approximation (GOA) to retrieve the particles refractive index from the localization of the photonic jet maxima, in addition to their size and 3D position. 


\section{Principle of the method and photonic jet reconstruction with DH}

The general principle of the method is depicted in Fig. 1(a) shows the direct calculation using LMT of the near-field intensity distribution of a spherical particle (radius $R=550 \mu \mathrm{m}$ and relative refractive index $m=m_{1} / m_{2}=1.0825$ ) illuminated by an unpolarized electromagnetic plane wave of wavelength $\lambda=\lambda_{0} / m_{2}=0.6328 \mu \mathrm{m}$, where $\lambda_{0}$ is the wavelength of the incident wave in a vacuum and $m_{1}=1.4425$ and $m_{2}=1.3325$ are the refractive indices of the particle and surrounding media for $\lambda_{0}$, respectively.

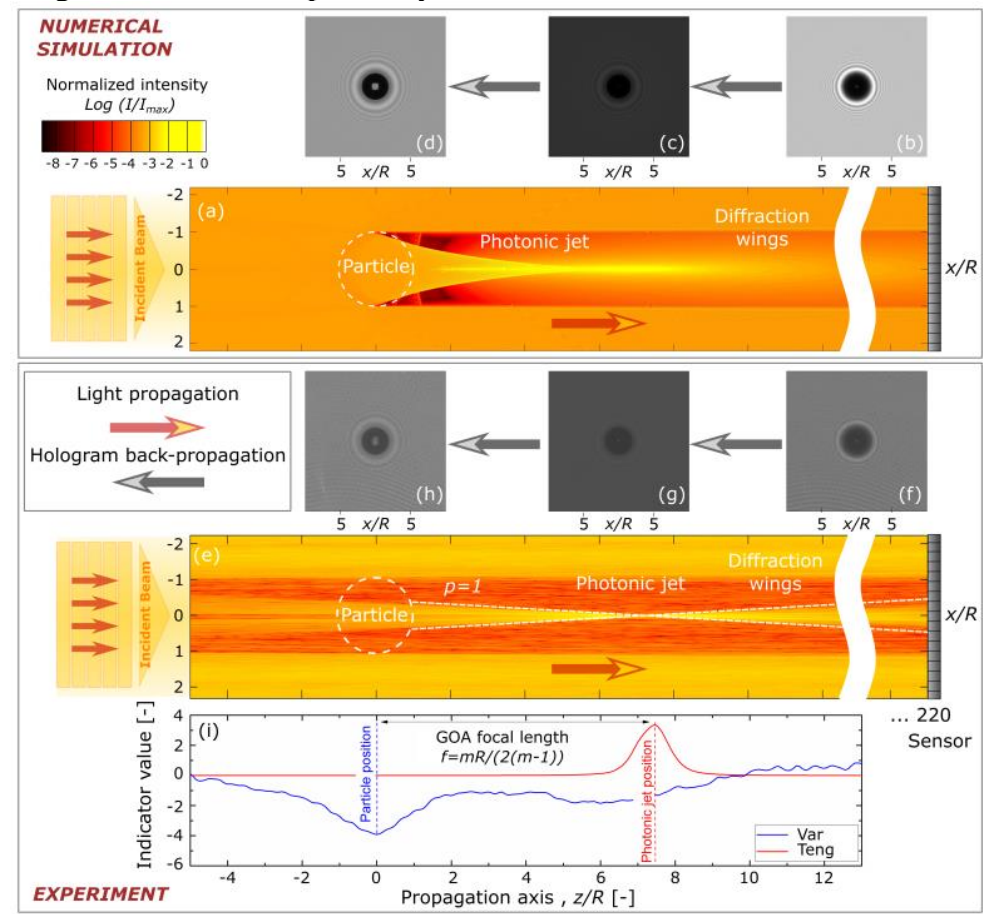

Fig. 1. Illustration of the proposed method to determine particle size, position, and refractive index using DH. (a) Direct LMT calculations of the near-field intensity distribution of a droplet with $R=550 \mu \mathrm{m}, m_{1}=1.4425$, and $m_{2}=1.3325$, (b) corresponding hologram in the sensor plane, and back-propagated holograms in the axial plane of (c) the photonic jet intensity maxima and (d) the particle center. (e)-(h) Similar to (a)-(d) but the hologram in (f) was produced by a real droplet with parameters similar to the droplet in (a). (i) Evolution of the indicators to determine the optimal reconstruction planes (see Visualization 1).

There are two specific regions of interest in Fig. 1(a): one is the forward diffraction, labeled "diffraction wings", and the second is a high-intensity and narrow region in the forward nearfield zone that corresponds to the focus of the photonic jet caustic [16]. Fig. 1(b) shows the hologram $I_{\mathrm{h}}$ that would be measured by a classical DH sensor (see Sec. 3 for details) located at the normalized distance $z_{s} / R=220$ (Fresnel number $F \geq 1$ [22]) from the particle center. From $I_{\mathrm{h}}$, the amplitude of the near-field, $U_{z}$, can be reconstructed in successive $z$-planes using a classical Fresnel deconvolution scheme [23]:

$$
U_{z}(x, y)=\left[I_{\mathrm{h}}(x, y)\right] *\left[\frac{1}{i \lambda z} \exp \left(\frac{2 i \pi z}{\lambda}\right) \exp \left(\frac{i \pi\left(x^{2}+y^{2}\right)}{\lambda z}\right)\right],
$$

where $i$ is the imaginary unit and the asterisk (*) indicates the convolution product. More details on the correction of Eq. (1) when interface and astigmatic effects occur are given in [22] and [13]. Back-propagations [22, 24] of this simulated hologram are shown in Fig. 1(c) for the axial position of the maximum intensity of the photonic jet, $z_{\max }$, and in Fig. 1(d) for the axial position of the particle center, $z_{\mathrm{p}}$. We compared the results of these calculations to those derived from 
Matthias P. L. Sentis, Fabrice R. A. Onofri, and Fabrice Lamadie, "Photonic jet reconstruction for particle refractive index measurement by digital in-line holography," Opt. Express 25, 867-873 (2017) https://doi.org/10.1364/OE.25.000867

an experiment performed using a droplet with similar parameters [see Fig. 1(e)-(h)]. Figure 1(e) shows the cross section of the modulus of the reconstructed near-field $U_{z}$, and Fig. 1(f) shows the experimentally recorded hologram. Clearly, only the coarse structures of the two regions of interest appear well restituted. To obtain $z_{\max }$ and $z_{\mathrm{p}}$ from $U_{z}$, two indicators are needed. By maximizing the Tenengrad variance of the modulus $\left|U_{z}\right|, z_{\max }$ can be accurately determined [13] [see Fig. 1(i)]. This estimator is calculated as follows:

$$
\text { Teng }(z)=\sum_{(x, y)}\left(\operatorname{Sob}\left[\left|U_{z}(x, y)\right|\right]-E\left\{\operatorname{Sob}\left[\left|U_{z}(x, y)\right|\right]\right\}\right)^{2},
$$

where Sob is the Sobel filter and $E(-)$ is the arithmetic mean. Similarly, $z_{\mathrm{p}}$ (and indirectly $x_{\mathrm{p}}, y_{\mathrm{p}}$, and $R$ ) can be obtained by minimizing the variance of the imaginary part of the reconstructed field, $\operatorname{Im}\left(U_{z}\right)[2]$ [see Fig. 1(h) and (i)]:

$$
\operatorname{Var}(z)=E\left(\operatorname{Im}\left[U_{z}(x, y)\right]-E\left\{\operatorname{Im}\left[U_{z}(x, y)\right]\right\}\right)^{2} .
$$

Finally, to determine the relative refractive index $m$ of the particle, we assumed that the particle is a ball-shaped lens with focal length $f(m, R)=\left|z_{\max }-z_{\mathrm{p}}\right|=m R / 2(m-1)[14,15,25]$. Thus, $m$ can be derived directly from the estimations of $z_{\max }$ and $z_{\mathrm{p}}$ :

$$
m \approx \frac{2\left|z_{\max }-z_{\mathrm{p}}\right|}{2\left|z_{\max }-z_{\mathrm{p}}\right|-R}
$$

The quality of the reconstruction of the particle near-field is decisive for the resolution of the proposed method and notably the region close to the photonic jet maxima because it is extremely localized (and thus strongly diverges) in the transverse direction. Therefore, this reconstruction is necessarily limited by the sampling conditions. Because most of the information on the photonic jet recorded by the sensor originates from the directly transmitted rays [ $p=1$ rays [26] as seen in Fig. 1(e)], there must be an optimum between the sensor plane position and its discretization step. Indeed, if the sensor plane is too close to the particle, all $p=1$ rays are collected but they might be undersampled, and vice versa.
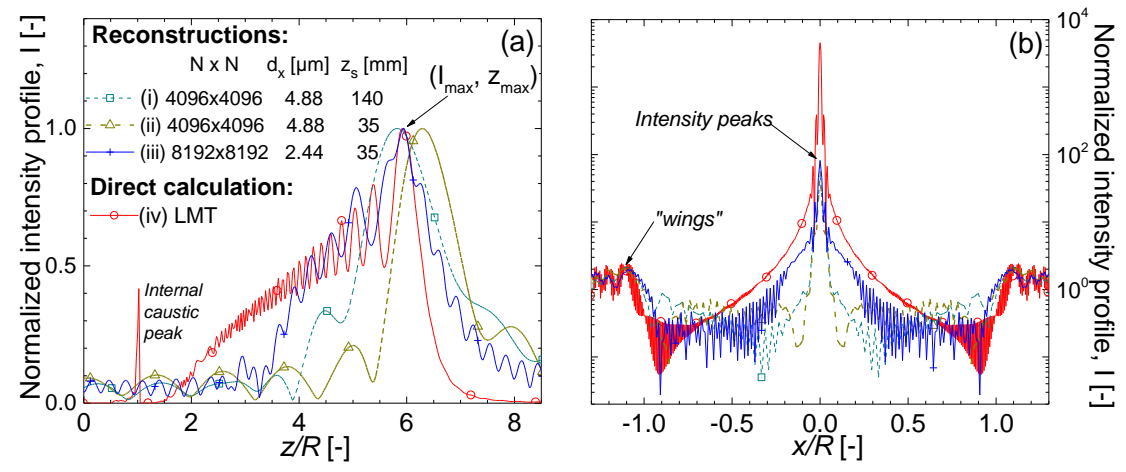

Fig. 2. Direct calculation and reconstruction of (a) axial and (b) transverse intensity profiles of the near-field region [droplets and other optical parameters the same as in Fig. 1(a) except for $R=250 \mu \mathrm{m}]$.

These effects are illustrated in Fig. 2 for (a) the axial $(z, x=y=0)$ and (b) the transverse $\left(z=z_{\max }, x, y=0\right)$ normalized intensity profiles of the near-field intensity distribution. Direct LMT calculations are compared with profiles reconstructed using Eq. (1) for three sampling conditions that are representative of high-resolution square sensors with $N \times N$ square pixels of $d x$ on a side. All other parameters are the same as those used in Fig. 1 except for the particle radius $(R=250 \mu \mathrm{m})$. Figure 2(a) clearly shows that the best reconstruction was obtained with configuration (iii), which had the largest angular field of view and angular resolution. Figure 
Matthias P. L. Sentis, Fabrice R. A. Onofri, and Fabrice Lamadie, "Photonic jet reconstruction for particle refractive index measurement by digital in-line holography," Opt. Express 25, 867-873 (2017) https://doi.org/10.1364/OE.25.000867

2(b) shows that $z_{\max }$ is perfectly retrieved and some high-frequency structures of the photonic jet are reconstructed (although not accurately) with configuration (iii). As expected, the degraded sampling conditions of configurations (i) and (ii) cause slight positive and negative shifts on $z_{\max }$.

\section{Numerical evaluation of the resolution and experimental validation}

The sensitivity of the method in terms of retrieval of particle parameters was evaluated first via numerical simulation. Holograms were generated for 1000 single droplets with uniformly distributed parameters: in space, with $x_{\mathrm{p}} \in[-6,6] \mathrm{mm}, y_{\mathrm{p}} \in[-5,5] \mathrm{mm}, z_{\mathrm{p}} \in[61,135] \mathrm{mm}$; in size, with $R \in[30,1250] \mu \mathrm{m}$; and in composition, with $m \in[1.07,1.6]$. These parameters were representative of various multiphase flows, including liquid-liquid systems (e.g., water droplets in various organic fluids, where $m \approx 1.08-1.25$ ) and solid particles or droplets in gas systems (e.g., water droplets or latex particles in air, $m \approx 1.33-1.6$ ). For these simulations, an Opaque and Phase Disk (OPD) model was preferred, because LMT calculations are very time consuming and it was recently shown [13] that the OPD model correctly predicts the position of the maximum intensity of the photonic jet. All other parameters were the same as those used in the experimental setup. Figure 3(a) compares the overall results obtained for the refractive indices with nominal values. Figure 3(b) shows the evolution of the dispersion defined at $1-\sigma_{m}$ (i.e., one standard deviation) for refractive index intervals of 0.02 . There is good agreement between the refractive indices obtained via simulation and the nominal values, i.e., little dispersion, for liquid-liquid systems, with an average standard deviation of $\sigma_{m} \approx 3.7 \times 10^{-3}$. However, for liquid-gas systems, the dispersion is greater, with $\sigma_{m}=1.6 \times 10^{-2}$. This difference in the expected resolution of the proposed method is explained by the behavior of the function $z_{\max }(m)$ [see the blue curve in Fig. 3(b)]. Figure 3 also shows that the resolution on $m$ is almost independent of the particle size in the considered range. However, note that, for this configuration and for droplets with radius below $30 \mu \mathrm{m}$, the resolution of the proposed method critically decreases (i.e. $p=1$ rays are too diverging).
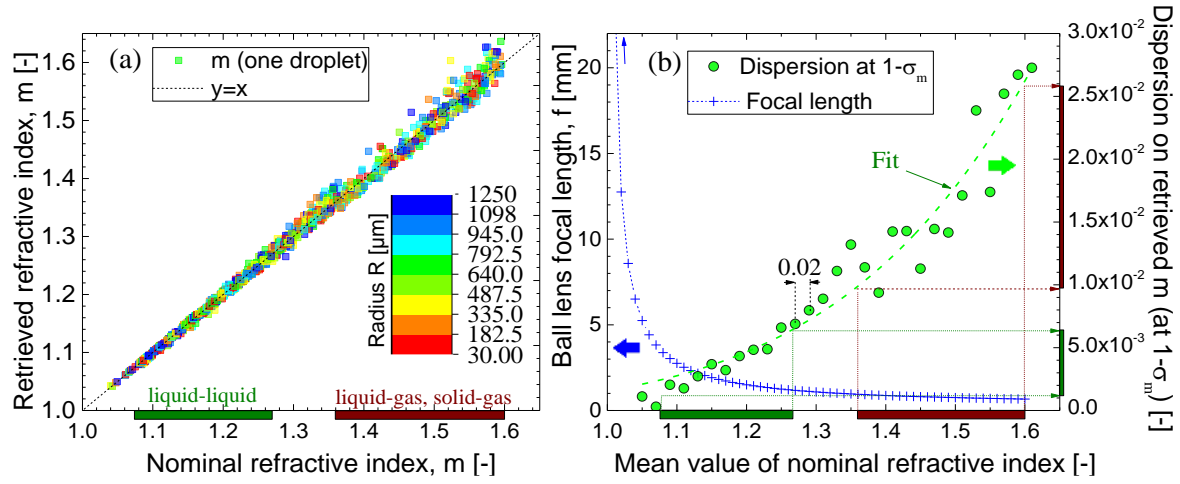

Fig. 3. Numerical evaluation of the resolution of the method with respect to the relative refractive index. (a) Holograms were simulated and processed for 1000 droplets with randomly distributed sizes, relative refractive indices, and 3D positions. (b) Evolution of the dispersion of the retrieved refractive index [data's were analyzed statistically over refractive index bandwidths of 0.02].

Next, we tested the method experimentally using a rectangular tank with glass walls and a $74 \times 74 \mathrm{~mm}^{2}$ horizontal section filled with demineralized water. The droplet generator, located at the center of the bottom of the tank, produced streams of monodisperse free-rising droplets $[13,22]$. Two pure liquids were used to generate the droplets: silicone oil $\left(m=1.0505 \pm 8 \times 10^{-4}\right)$ and Isane $\left(m=1.0657 \pm 8 \times 10^{-4}\right)$, as well as a 50/50 mixture in volume of Isane and Marcol $\left(m=1.0826 \pm 8 \times 10^{-4}\right)$. A benchtop Abbe refractometer was used to measure the refractive indices of these liquids. The uncertainty of $\pm 8 \times 10^{-4}$ was calculated from the estimated variations in temperature $\left( \pm 2{ }^{\circ} \mathrm{C}\right)$ during the course of the different experiments. The DH setup comprised a $5 \mathrm{~mW}$ unpolarized red HeNe laser injected into a fiber and 
Matthias P. L. Sentis, Fabrice R. A. Onofri, and Fabrice Lamadie, "Photonic jet reconstruction for particle refractive index measurement by digital in-line holography," Opt. Express 25, 867-873 (2017) https://doi.org/10.1364/OE.25.000867

collimated by magnification optics [1]. Signal acquisition was performed by a SCMOS camera $\left(\mathrm{PCO}^{\circledR}\right.$ Edge, 12 bits, $N \times N=2560 \times 2160, d x=6.5 \mu \mathrm{m}$ ) placed $40 \mathrm{~cm}$ above the droplet generator and $z_{s}=100 \mathrm{~mm}$ from the center of the tank where the droplets passed. For the reconstructions, the probed volume ( $74 \mathrm{~mm}$ of depth) is sliced along $\mathrm{z}$-axis in 1480 steps (i.e. $50 \mu \mathrm{m}$ increment). The whole reconstruction process takes about 5 min with Matlab ${ }^{\circledR}$ (without any particular optimization) on a desktop computer.

Figure 4 shows the statistical results obtained for (a) the refractive indices and (b) the sizes of three sets of 500 droplets. The Gaussian fit is added to guide the eye of the reader. Mean refractive indices are found to be in good agreement with the expected values, and the experimental dispersion $\sigma_{m}$ of the refractive index for Isane is low but slightly larger than that for the silicone oil. These two results agree well with the numerical study and the results shown in Fig. 3. Conversely, the abnormal dispersion of the refractive index of the mixed solution cannot be attributed to the decrease in sensitivity of the method that occurs with an increase in relative refractive index. The demixing of the Isane and Marcol mixture induced this abnormal dispersion. The abnormal dispersion demonstrates the ability of the method to detect some inhomogeneities within the droplets, which shows the possibility of using the method for reactive flow studies (e.g., precipitation or crystallization) [27]. Figure 4(b) compares the droplet sizes retrieved with DH with respect to the droplet count with those expected from the operating conditions of the droplet generator. The agreement between the two is very convincing.
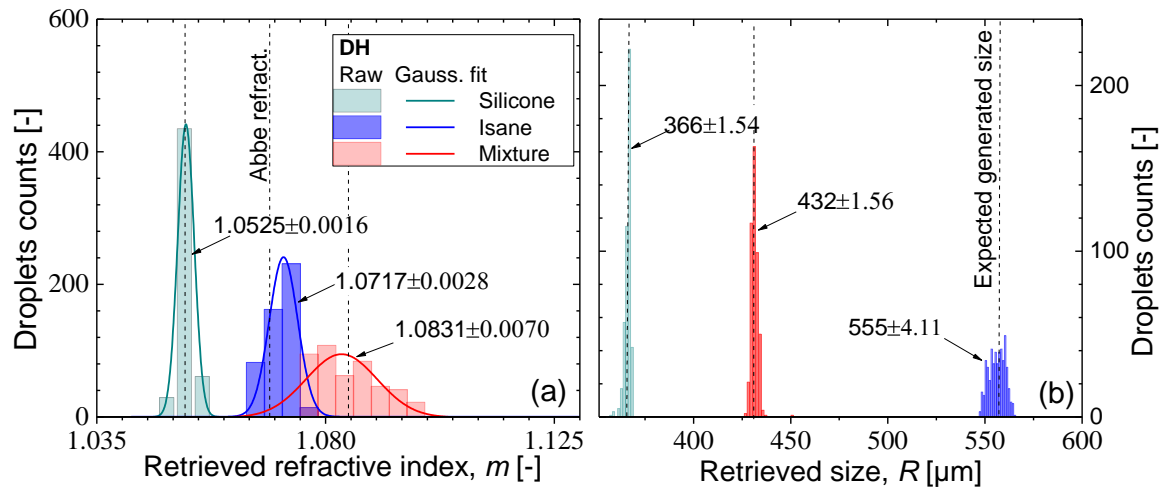

Fig. 4. Estimation of (a) the refractive index $m$ and (b) the radii $R$ for three series of 500 quasimonodisperse droplets of different liquids (two pure liquids and a non homogeneous mixture).

\section{Conclusion}

A novel method was proposed to determine the refractive index, size, and 3D position of spherical and transparent particles using $\mathrm{DH}$, based on the distance between the particle center and its photonic jet intensity maxima, retrieved from back-propagated holograms. Numerical simulations and experimental results for single liquid-liquid droplets showed that a resolution of $\left\langle 4 \times 10^{-3}\right.$ for the refractive index is achievable for particles with $R>30 \mu \mathrm{m}$, showing the possibility of using the method for investigating multiphase flows. For solid or liquid particles in a gas, the resolution of the refractive index is lower (numerical estimation: $1.6 \times 10^{-2}$ ), but could be sufficient for recognizing particle material. The application of this method for characterization of more complex flows should be straightforward.

\section{Funding}

Nuclear Energy Division of the CEA (DISN/PAREC); French National Research Agency (grant ANR-13-BS09-0008-02). 